\title{
Efeito da bagana de carnaúba nos atributos microbiológicos, umidade e temperatura do solo
}

\section{Effect of carnauba bagana on microbiological attributes, moisture and temperature of soil}

\section{Célia Ribeiro do NASCIMENTO'; Anderson Chaves RODRIGUES ${ }^{2}$; Francineuma Ponciano de ARRUDA $^{3}$; Ricardo Silva de SOUSA ${ }^{4}$; Luís Alfredo Pinheiro Leal NUNES ${ }^{5}$}

\footnotetext{
${ }_{1}^{1}$ Mestre em Agronomia pela Universidade Federal do Piauí (UFPI), celiaribeironascimento@gmail.com

${ }^{2}$ Mestrando no Programa de Pós-graduação em Agronomia, UFPI, andersonrodrigues88@hotmail.com

${ }^{3}$ Doutora em Solos e Nutrição de Planta, UFPB, Docente do Centro de Ciências Agrárias da Universidade Estadual do Piauí, Teresina-PI, neuma-arruda@hotmail.com

4 Doutor em Agronomia (Agricultura Tropical) - UFPI, Docente do Departamento de Engenharia Agrícola e Solos pela Universidade Federal do Piauí, Teresina-PI, ricardoss@ufpi.edu.br

${ }^{5}$ Autor para Correspondência: Doutor em Solos e Nutrição de Plantas (UFV), Docente do Departamento de Engenharia Agrícola e Solos pela Universidade Federal do Piauí, Teresina-PI, luisalfredo@ufpi.edu.br
}

\section{Recebido em: 22-07-2021; Aceito em: 13-01-2022}

\section{Resumo}

O resíduo resultante da extração da cera da folha da carnaúba, conhecido como bagana de carnaúba, e ocasionalmente é utilizado como adubo orgânico, mas pouco se conhece o melhor modo de aplicação e sua relação com adubação mineral. O objetivo do trabalho foi verificar o efeito da aplicação superficial e a incorporação da bagana de carnaúba associada a adubação NPK sobre os atributos microbiológicos, temperatura e umidade do solo. O delineamento experimental foi em bloco casualizado constituídos de seis tratamentos e quatro repetições para plantio de milho (Zea mays). Os tratamentos foram: a) aplicação superficial de $8 \mathrm{Mg} \mathrm{ha}^{-1}$ bagana de carnaúba; b) incorporação de $8 \mathrm{Mg} \mathrm{ha}^{-1}$ bagana de carnaúba; c) aplicação superficial de $8 \mathrm{Mg} \mathrm{ha}^{-1}$ bagana de carnaúba + NPK, d) incorporação de $8 \mathrm{Mg} \mathrm{ha}^{-1}$ bagana de carnaúba + NPK, e) Aplicação de NPK e, e) controle onde não ocorreu qualquer adubação. Foram coletadas amostras do solo das parcelas, após colheita do milho, para avaliar o carbono da biomassa microbiana (CBM); respiração basal (RB); quociente microbiano (qMIC), quociente metabólico $\left(\mathrm{qCO}_{2}\right)$ e o carbono orgânico total (COT). Durante o experimento foram realizadas coletas de solo para determinação da umidade e temperatura. Os tratamentos que receberam adição de bagana de carnaúba indicam um maior incremento de COT. A aplicação da bagana de carnaúba em superfície associada a adubação NPK contribuiu com o aumento das médias do CBM e qMIC. Os tratamentos com incorporação de $8 \mathrm{Mg} \mathrm{ha}^{-1}$ de bagana de carnaúba e testemunha evidenciaram um maior quociente metabólico. A bagana da carnaúba aplicada na superfície do solo contribuiu para a manutenção de uma maior umidade e menor temperatura no solo. Por meio de análise multivariadas, foi possível verificar que a utilização bagana de carnaúba em superfície isolado ou associado ao NPK mostraram os melhores resultados com relação ao carbono microbiano, carbono orgânico e umidade do solo.

Palavras-chave adicionais: adubação orgânica; carbono microbiano; Copernicia prunifera; resíduo agroindustrial.

\begin{abstract}
The waste resulting from the extraction of wax from the carnauba leaf, known as carnauba bagana, is occasionally used as organic fertilizer, but little is known about the best way of application and its relationship with mineral fertilizer. The objective of this work was to verify the effect of surface application and incorporation of carnauba bagana associated with NPK fertilization on microbiological attributes, temperature and soil moisture. The experimental design was a randomized block consisting of six treatments and four replications for planting corn (Zea mays). The treatments were: a) superficial application of $8 \mathrm{Mg} \mathrm{ha}^{-1}$ carnauba bagana; b) incorporation of $8 \mathrm{Mg} \mathrm{ha}^{-1}$ carnauba bagana; c) surface application of $8 \mathrm{Mg} \mathrm{ha}^{-1}$ carnauba bagana + NPK, d) incorporation of 8 $\mathrm{Mg} \mathrm{ha}^{-1}$ carnauba bagana + NPK, e) NPK application and, e) control where no fertilization occurred. Soil samples were collected from the plots, after corn harvest, to evaluate the microbial biomass carbon (MBC); basal respiration (BR); microbial quotient ( $\mathrm{qMIC})$, metabolic quotient $\left(\mathrm{qCO}_{2}\right)$ and total organic carbon (TOC). During the experiment, soil samples were collected to determine moisture and temperature. The treatments that received the addition of carnauba bagana indicate a greater increase in TOC. The application of carnauba bagana on the surface associated with NPK fertilization contributed to the increase of the averages of MBC and qMIC. The treatments with incorporation of $8 \mathrm{Mg} \mathrm{ha}^{-1}$ of carnauba bagana and control showed a higher metabolic quotient. The carnauba bagana applied to the soil surface contributed to the maintenance of higher moisture and lower temperature in the soil. Through multivariate analysis, it was possible to verify that the use of carnauba bagana on the surface alone or associated with NPK showed the best results in relation to microbial carbon, organic carbon and soil moisture.
\end{abstract}

Additional keywords: agroindustrial waste; Copernicia prunifera; microbial carbono; organic fertilizer. 


\section{Introdução}

A carnaúba Copernicia prunifera (Miller) H. E. Moore é uma palmeira da família Arecaceae, nativa do Brasil e endêmica do semiárido do Nordeste, distribuem-se principalmente nos estados do Piauí, Ceará e Rio Grande do Norte (Sousa et al., 2015). A bagana de carnaúba é um subproduto da extração de cera da palha dessa palmeira, com característica de baixa decomposição, mas que apresenta grande potencial de reaproveitamento como cobertura morta do solo (Silva et al., 2018). A cobertura cerífera presente no resíduo reflete a radiação solar, funcionando como o agente protetor contra a radiação, suportando até 3000 horas de insolação por ano (Jetter \& Kunst, 2008).

A bagana de carnaúba é um material rico em frações de carbono de maior densidade tais como os compostos ligninícos e os polifenóis, que possuem cadeias carbônicas complexas, o que torna a bagana um resíduo com elevada relação $\mathrm{C} / \mathrm{N}$ (Gomes et. al. 2009). Pesquisa realizada por Oliveira et al. (2018) mostraram que a bagana de carnaúba tem valores elevados de carbono orgânico e de alguns nutrientes essenciais para as plantas, como o cálcio e o ferro.

A bagana de carnaúba, além de servir como incremento da matéria orgânica (Gonçalves et al., 2019), atua na manutenção de umidade do solo para as raízes, podendo assim prolongar o tempo de disponibilidade de água sobretudo em períodos de estiagem prolongada (Gonçalves et al., 2020), e atenuação da amplitude térmica (Nogueira et al., 2015), o que favorece a atividade microbiana no solo (Ziech et al., 2014).

Vários trabalhos realizados com substratos orgânicos de bagana de carnaúba têm mostrado resultados promissores em relação a manutenção de uma maior umidade no solo. Estudo utilizando diferentes materiais como cobertura morta do solo no cultivo de pimentão verificou que o diâmetro de frutos apresentou maior valor com o uso da bagana de carnaúba em função de uma maior umidade do solo e menor incidência de ervas daninhas, proporcionando, desta maneira, melhor desenvolvimento dos frutos (Queiroga et al., 2002). De acordo com Sousa et al., (2017), o uso de palha de carnaúba como cobertura do solo reflete parte da energia solar que sobre ela incide, reduzindo consideravelmente as trocas gasosas entre o solo e a atmosfera, proporcionando assim menor perda de umidade do solo, o que maximiza o uso da água pelas plantas.

A manutenção ou incorporação de resíduos orgânicos na superfície do solo, forma uma camada de material vegetal em diferentes estágios de decomposição, proporcionando fonte de energia e microclima com menor variação térmica e maior disponibilidade de água favorecendo 0 estabelecimento e atividade de microrganismos (Duarte et el., 2014). A microbiota do solo é a principal responsável pela decomposição dos resíduos orgânicos, pela ciclagem de nutrientes e pelo fluxo de energia no solo, podendo ter influência tanto na transformação da matéria orgânica, quanto na estocagem do carbono e nutrientes minerais (Moreira \& Siqueira, 2006).

Os indicadores biológicos do solo, que incluem a biomassa microbiana, a respiração microbiana, a atividade enzimática e o quociente metabólico, são bastante utilizados para o monitoramento da qualidade do solo no que se refere não apenas à ciclagem dos nutrientes, como também na estimativa da capacidade do solo para o crescimento vegetal (Araújo et al. 2007). A biomassa microbiana é a parte ativa da matéria orgânica do solo, e constitui-se num indicador de qualidade largamente utilizado para a averiguação de pequenas mudanças impostas pelas alterações no uso e manejo empregados neste sistema. Alterações nessa variável reflete na quantidade e qualidade das fontes de carbono existentes ou adicionadas ao solo (Wang et al., 2020, Araújo, Melo, 2010).

Baseado nessas informações, o objetivo deste trabalho foi verificar o impacto da aplicação superficial e incorporada da bagana de carnaúba sobre os atributos microbiológicos, umidade e temperatura do solo.

\section{Material e métodos}

O experimento foi realizado na área experimental do Colégio Técnico de Teresina (CTT), localizado na cidade de Teresina, Piauí $\left(5^{\circ} 2^{\prime} 58,48^{\prime \prime}\right.$ S; $42^{\circ} 46^{\prime} 57,13^{\prime \prime O}$ e altitude $86 \mathrm{~m}$ ). A classificação climática da região é definida como subúmido seco, apresentando precipitação pluvial média de 1.342,4 $\mathrm{mm}$ ano-1 com média anual de temperatura do ar máxima, mínima e média de 33,$9 ; 22,3 ; 28,1{ }^{\circ} \mathrm{C}$, respectivamente e umidade relativa do ar e evapotranspiração de referência anual média de $69,6 \%$ e $4,7 \mathrm{~mm} \mathrm{dia}^{-1}$, respectivamente. O período chuvoso se estende de dezembro a maio, e os meses de fevereiro, março e abril formam o trimestre mais úmido do ano.

O solo foi classificado como Latossolo Vermelho Amarelo distrófico com $800 \mathrm{~g} \mathrm{~kg}^{-1}$ de areia, $110 \mathrm{~g} \mathrm{~kg}^{-1}$ de silte e $90 \mathrm{~g} \mathrm{~kg}^{-1}$ de argila, caracterizando uma textura franco arenosa. A análise química do solo encontra-se na tabela 1 . Verifica-se que os valores de $\mathrm{P}$ e K mostraram-se baixos o que justifica a necessidade de adubação desse solo. A área do experimento já vinha sendo utilizadas com culturas anuais há cerca de 10 anos. 
Tabela 1 - Características químicas do solo na profundidade de $0-20 \mathrm{~cm}$ da área experimental do Colégio Técnico de Teresina (CTT). Soil chemical characteristics at depth of $0-20 \mathrm{~cm}$ from the experimental área of the Technical College of Teresina (CTT).

\begin{tabular}{ccccccccccc}
\hline $\mathrm{pH}$ & $\mathrm{MO}$ & $\mathrm{P}$ & $\mathrm{K}$ & $\mathrm{Ca}$ & $\mathrm{Mg}$ & $\mathrm{Al}$ & $\mathrm{H}+\mathrm{Al}$ & $\mathrm{CTC}$ & $\mathrm{SB}$ & $\mathrm{V}$ \\
\hline & $\mathrm{g} \mathrm{kg}^{-1}$ & $\mathrm{mg} \mathrm{dm}^{-3}$ & - & - & & & & \\
\hline 4,3 & 9,8 & 8 & 0,12 & 0,95 & 0,25 & 0,19 & 1,87 & 3,19 & 1,32 & 41,4 \\
\hline
\end{tabular}

Foi adotado um delineamento experimental foi em blocos casualizados com seis tratamentos e quatro repetições para semeadura do milho. A aplicação da bagana de carnaúba foi feita 30 dias antes da semeadura na superfície do solo e incorporado com uma enxada a $20 \mathrm{~cm}$ de profundidade. A semeadura ocorreu no mês de fevereiro e a colheita foi realizada após 71 dias. Os tratamentos consistiram em: a) Aplicação de $8 \mathrm{Mg} \mathrm{ha}^{-1}$ bagana de carnaúba na superfície do solo (BS); b) Aplicação de $8 \mathrm{Mg} \mathrm{ha}^{-1}$ bagana de carnaúba incorporada ao solo (BI); c) Aplicação de $8 \mathrm{Mg} \mathrm{ha}^{-1}$ bagana de carnaúba na superfície do solo + (BS + NPK), d) Aplicação de 8 Mg ha $^{-1}$ bagana de carnaúba incorporada ao solo + NPK (BI + NPK, e) aplicação de NPK e f) Testemunha sem aplicação de adubo mineral ou bagana de carnaúba. Os valores de NPK foram $100 \mathrm{~kg} \mathrm{ha}^{-1}$ de sulfato de amônio, $180 \mathrm{~kg} \mathrm{ha}^{-1}$ superfosfato triplo e $86 \mathrm{~kg} \mathrm{ha}^{-1} \mathrm{de}$ cloreto de potássio, definidos em função da análise química do solo, conforme Avarez et al, (1999). As parcelas foram de $2 \times 5 \mathrm{~m}$ e um espaço de $1 \mathrm{~m}$ entre elas. O espaçamento utilizado foi de $0,40 \times 0,33 \mathrm{~m}$ sendo a cultivar utilizada a AG 1051, híbrido simples, de ciclo semiprecoce, com população de 75.000 plantas por hectare.

Após a colheita do milho foram coletadas amostras do solo nas entrelinhas para análises microbiológicas e de carbono orgânico em cada parcela num total de quatro amostras simples nas profundidades de $0-0,20 \mathrm{~m}$ para formar uma amostra composta. As amostras para análise microbiológicas foram identificadas e acondicionadas em sacos plásticos com respirador e transportadas em caixa de isopor até o laboratório e colocadas em refrigeração a $4^{\circ} \mathrm{C}$.

O carbono da biomassa microbiana (CBM) foi determinado pelo método da irradiação-extração (Islam \& Weil, 1998). A partir dos valores do CBM e do conteúdo de COT (Carbono orgânico total), foi calculado o quociente microbiano (qMIC) por meio da seguinte expressão: qMIC $=$ CBM $/$ COT $\times 100$. O carbono orgânico (CO) foi analisado em via úmida, segundo o método proposto por Yeomans \& Bremner (1988).

A respiração do solo foi estimada pela quantificação do $\mathrm{CO}_{2}$ liberado durante sete dias de incubação do solo em sistema fechado. $\mathrm{O} \quad \mathrm{CO}_{2}$ produzido foi capturado em solução de $\mathrm{NaOH}$ (1 mol L' $\left.{ }^{1}\right)$ e posteriormente titulado com $\mathrm{HCL}\left(0,05 \mathrm{~mol} \mathrm{~L}^{-1}\right)$. O quociente respiratório que representa a respiração microbiana por unidade de biomassa foi determinado $\mathrm{e}$ expresso em $\mathrm{mg} \mathrm{CCO}_{2} \mathrm{~g} \mathrm{Cmic.}^{-1}$.

A temperatura da superfície do solo foi medida em ${ }^{0} \mathrm{C}$ com o auxílio de um termômetro digital. Para a determinação da umidade do solo foram realizadas coletas nas parcelas, na profundidade de 0,0-0,20 m. Para avaliação da umidade as amostras foram submetidas ao método termogravimétrico, que consiste em pesar o solo úmido (MU) e em seguida secá-lo em estufa a $105-110^{\circ} \mathrm{C}$ por 24 horas e, após esse período, determinar sua massa seca (MS). A umidade foi calculada pela equação: $U=\mathrm{Mu}-\mathrm{Ms} / \mathrm{Mu} \times 100 \mathrm{em}$ que: $\mathrm{U}=$ umidade do solo $(\mathrm{kg} \mathrm{kg}-1)$; $\mathrm{Mu}=$ massa de solo úmido (g); Ms = massa de solo seco (g).

Inicialmente, os dados foram analisados pelo teste de Shapiro-Wilk para avaliar a normalidade. Em seguida, submetidos à análise de variância precedida pelo teste $\mathrm{F}$, sendo considerado um nível mínimo de significância de $5 \%$ de probabilidade. Para comparar as médias de cada tratamento, foi utilizado o teste Scott-Knott $(p<0,05)$. Foram realizadas também técnicas multivariadas de Análises de Componentes Principais (ACP) para o entendimento de como as variáveis interagiram ao mesmo tempo.

Como os dados são expressos em unidades de medidas diferentes, estes foram padronizados para assegurar que todas as variáveis contribuam igualmente para o modelo independente da escala. Todas as análises foram processadas no programa $R$ (R Core Team 2016).

\section{Resultados e discussão}

Os tratamentos que receberam adição de bagana de carnaúba mostraram um maior incremento de carbono orgânico total (COT) em relação a testemunha (Figura 1). Estudos realizados por Oliveira et al. (2018) revelaram que a análise da composição química da bagana de carnaúba mostrou teor de carbono orgânico de $161,6 \mathrm{~g} \mathrm{~kg}^{-1}$ que é considerado alto. Ainda segundo os mesmos autores, de acordo com a Instrução Normativa DAS/MAPA 25/2009, esse resíduo apresenta teores de nutrientes que permitem que os mesmos sejam usados como adubos orgânicos. 

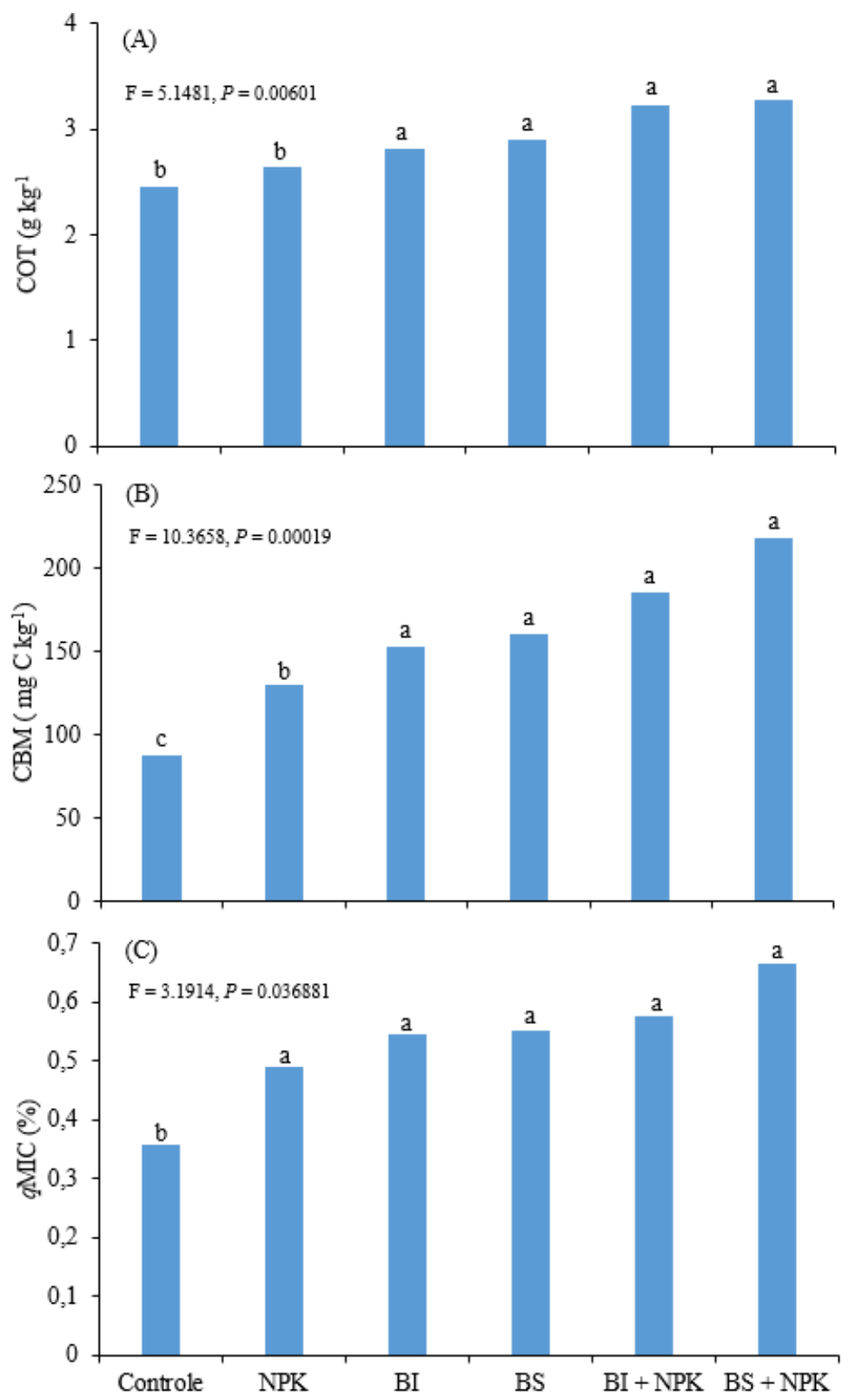

Figura 1 - Carbono orgânico total, COT (A), carbono da biomassa microbiana, CBM (B) e quociente microbiano, qMIC (C) em solo com bagana de carnaúba em cobertura ou incorporada e associada ou não a NPK em referência ao solo descoberto e não fertilizado (controle) e uso de adubação mineral com NPK. BI: bagana incorporada; BS: bagana em superfície; todos os tratamentos com bagana receberam $8 \mathrm{t} \mathrm{ha}^{-1}$. Total organic carbon (A), microbial biomass carbon $(B)$ and microbial quotient $(C)$ in soil cultivated under covered or incorporated carnauba berry and associated or not with NPK in reference to bare and unfertilized soil (control) and use of mineral fertilizer with NPK.

* Letras iguais nas barras não diferem entre si pelo teste de Scott-Knott $(P<0,05)$.

De acordo com Santoja et al. (2015), quando a relação C / N no resíduo orgânico é superior a 30 , existe inicialmente predomínio da imobilização de nutrientes e o tempo de decomposição só se intensifica depois dos 60 dias. Pesquisa realizada por Souza et al. (2018) concluiu que a cobertura morta com palha de sorgo com alta relação $\mathrm{C} / \mathrm{N}$ aplicada no solo foi eficiente em aumentar o teor de carbono orgânico do solo durante os quatro meses do ciclo da cultura, resultados semelhantes ao estudado.

Em geral os resultados da CBM e qMIC mostraram um incremento nos tratamentos com adição de bagana de carnaúba isolada ou associados com NPK. Como o CBM é reconhecida como um indicador chave da qualidade do solo (Araújo \& Monteiro, 2007), esses resultados sugerem que aplicação desse resíduo disponibiliza substrato aos 
microrganismos do solo mesmo permitindo uso de fontes de $\mathrm{C}$ pelos microrganismos do solo e, assim, aumentar o qMIC. Em manejos com maior deposição de resíduos orgânicos no solo, há estímulo da biomassa microbiana, o que acarreta seu aumento populacional e sua atividade, visto que além de aumentar a disponibilidade de substrato, determina condições de menor variação térmica e maior disponibilidade de água (Bonetti et al., 2018).
O tratamento $\mathrm{Bl}$ mostrou os melhores resultados para a respiração do solo seguido de BS, o que poderia indicar um maior equilíbrio energético nesses sistemas (Figura 2). No entanto, uma maior respiração do solo pode indicar estresse ecológico e menor eficiência de uso de carbono (Araújo et al. 2010). Para fins de comprovação, avaliamos o quociente metabólico $\left(\mathrm{qCO}_{2}\right)$, que é um indicador útil da atividade da biomassa microbiana.

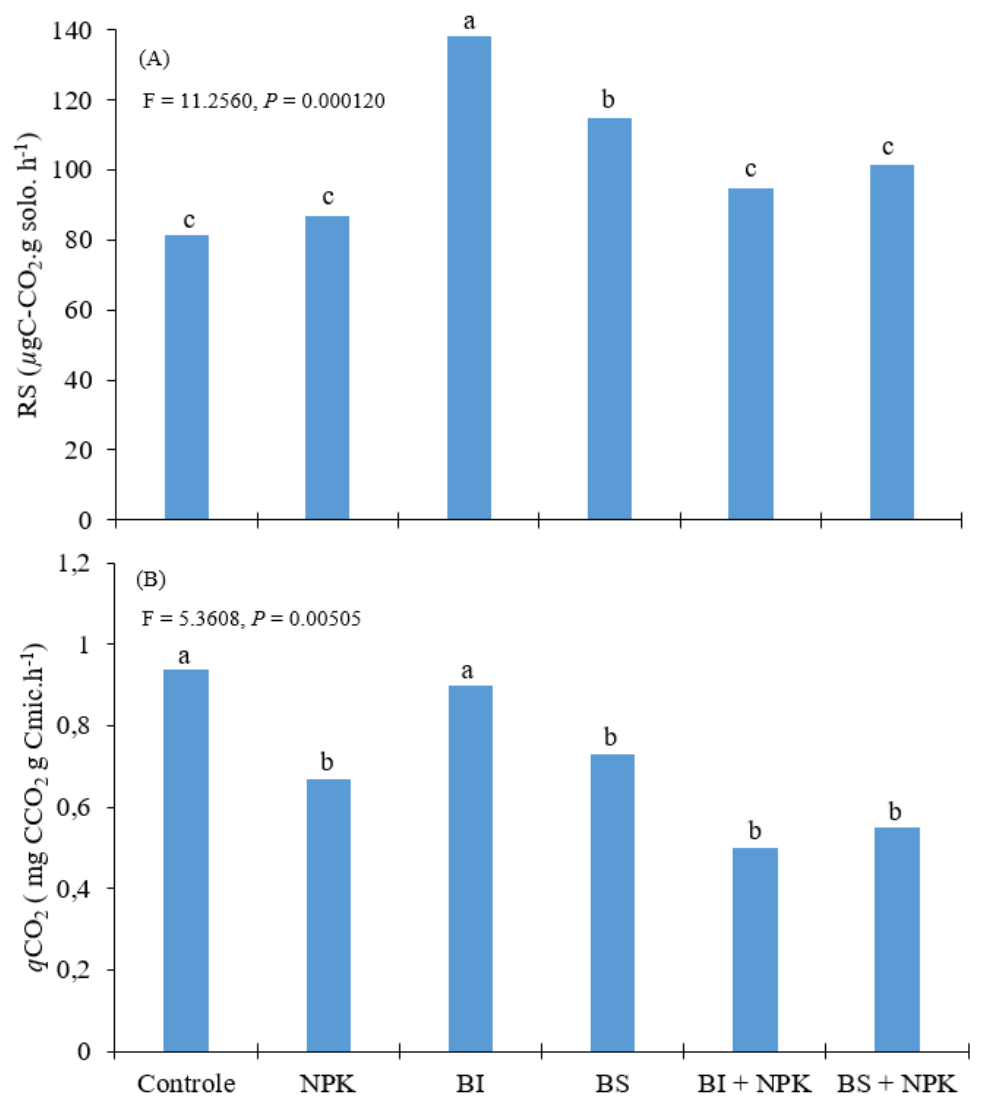

Figura 2 - Respiração, RS (A) e quociente metabólico, $q \mathrm{CO}_{2}$ (B) em solo com bagana de carnaúba em cobertura ou incorporada e associada ou não a NPK em referência ao solo descoberto e não fertilizado (controle) e uso de adubação mineral com NPK. BI: bagana incorporada; BS: bagana em superfície; todos os tratamentos com bagana receberam $8 \mathrm{t} \mathrm{ha}^{-1}$. Letras iguais nas barras não diferem entre si pelo teste de Scott-Knott $(P<0,05)$. Soil respiration $(A)$ and metabolic quotient $(B)$ in soil cultivated under covered or incorporated carnauba berry and associated or not with NPK in reference to bare and unfertilized soil (control) and use of mineral fertilizer with NPK.

Os tratamentos com NPK isolado ou associados a bagana de carnaúba mostraram menores valores de $\mathrm{qCO}_{2}$, o que indica uma maior eficiência energética no uso do $C$ pelos microrganismos. Por outro lado, o tratamento BCINC e o controle mostraram maiores valores dessa variável que simboliza uma biomassa menos eficiente que perde mais $\mathrm{C}$ na forma de $\mathrm{CO}_{2}$, e incorpora menos em seus tecidos.

A maior relação $\mathrm{C} / \mathrm{N}$ da bagana de carnaúba aliada a incorporação do resíduo ao solo provavelmente não favoreceu a decomposição do resíduo. Resíduos com alta relação $\mathrm{C} / \mathrm{N}$ e quando incorporados ao solo não tem quantidades de $\mathrm{N}$ suficientes para a formação de todos os componentes celulares, o que faz com que parte desse nitrogênio tenha que ser imobilizado do solo, demandando investimentos maiores de energia, pois uma maior quantidade de enzimas captadoras desse nutriente é sintetizada (Brady \& Weil, 2013). Pesquisa realizada por Giacomini et al., (2008) concluíram que incorporação da palha de aveia no solo proporcional uma menor mineralização do $\mathrm{C}$ desses materiais 
orgânicos, quando comparada à sua manutenção na superfície.

$\mathrm{Na}$ figura 3 encontram-se os valores de temperatura e umidade dos tratamentos durante a condução do experimento, observa-se que os tratamentos que receberam a bagana de carnaúba mostraram uma menor temperatura e maior umidade ao longo do experimento. Por outro lado, os tratamentos sem nenhuma cobertura mostraram menores valores nessas variáveis provavelmente em função da textura arenosa desse solo e da ausência do resíduo para atenuar os efeitos da radiação solar.
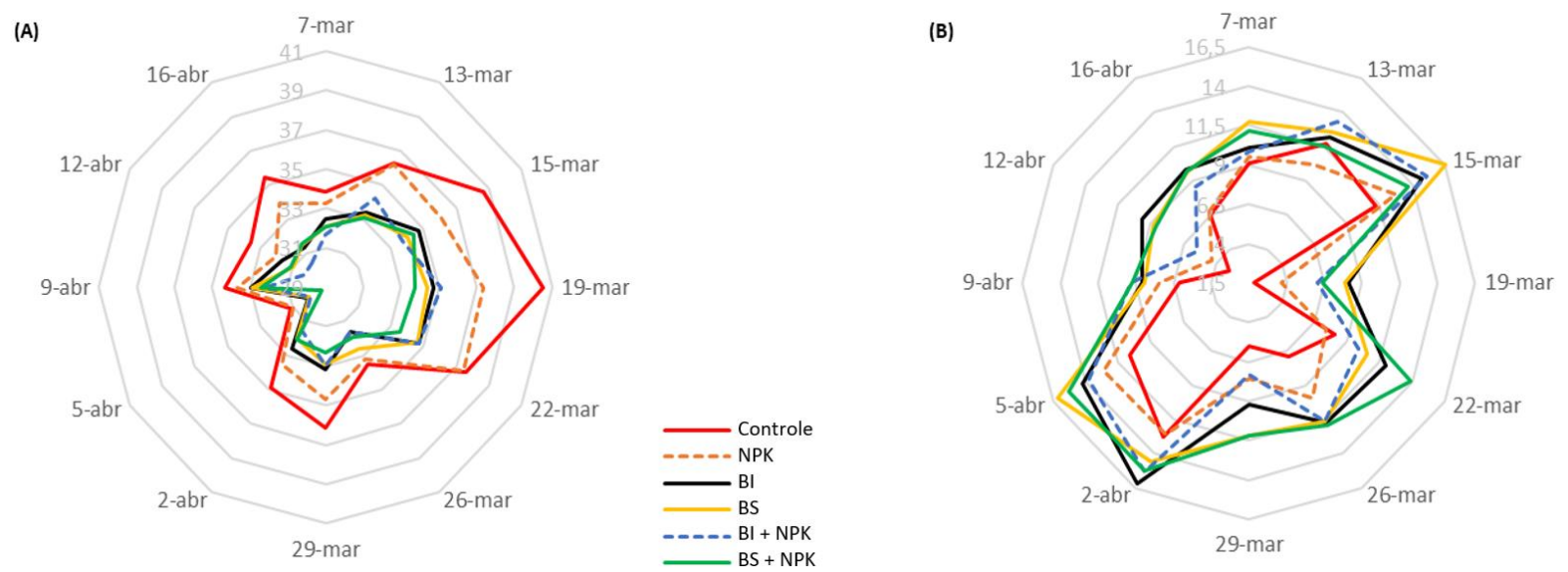

Figura 3 - Médias de temperatura (A) e umidade do solo (B) sob bagana de carnaúba em cobertura ou incorporada e associada ou não a NPK em referência ao solo descoberto e não fertilizado (controle) e uso de adubação mineral com NPK. A linha cinza clara mostra escala de variação média das variáveis. Bl: bagana incorporada; BS: bagana em superfície; todos os tratamentos com bagana receberam $8 \mathrm{t} \mathrm{ha}^{-1}$. Average temperature $(A)$ and soil moisture $(B)$ under covered or incorporated carnauba bagasse and associated or not with NPK in reference to uncovered and unfertilized soil (control) and use of mineral fertilizer with NPK. The light gray line shows the average variation scale of the variables.

Nos estádios iniciais da cultura do milho, os valores de temperatura média do solo foram elevados nos tratamentos sem cobertura da bagana, isso porque neste período o índice de área foliar é baixo, logo o solo recebia mais energia solar direta. Os dados demostram que a cobertura atuou como isolante

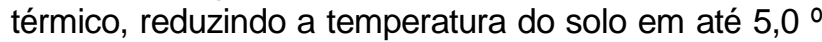
$\mathrm{C}$, no tratamento BS. Avaliando a temperatura do solo sem cobertura e com cobertura de 4 e $8 \mathrm{t} \mathrm{ha}^{-1}$ de palhada de aveia, Vieira et al. (2020) também observaram redução da temperatura do solo (de até 2,9 ${ }^{\circ} \mathrm{C}$ ), quando se utilizou $8 \mathrm{t} \mathrm{ha}^{-1}$ de cobertura de palhada de braquiária sobre o solo. Já Gasparim et al. (2005) verificaram que a aplicação de $8 \mathrm{t} \mathrm{ha}^{-1}$ de aveia preta resultou em uma menor amplitude de variação da temperatura do solo em cerca de $3^{0} \mathrm{C}$.

Pesquisa realizada num solo de textura arenosa com a utilização de cobertura morta com $16 \mathrm{t}$ ha $^{-1}$ bagana de carnaúba, concluiu que esse resíduo potencializou os níveis de produtividade da água de irrigação na cultura do mamoeiro (Sousa et al., 2017). Ademais, Souza et al. (2016) mostraram que o uso de cobertura de solo com a bagana de carnaúba, foi eficiente em reter água no solo, como também na inibição total do surgimento de plantas daninhas no desenvolvimento inicial do alface.

Gonçalves et al. (2020) afirmam ser a bagana de carnaúba é um importante insumo para o semiárido nordestino, caracterizado pelas baixas precipitações e elevadas temperaturas, principalmente devido aos seus efeitos sobre 0 aumento na eficiência de irrigação, decorrente da redução na evaporação de água. Portanto, a maior capacidade de retenção de água pela bagana de carnaúba o caracteriza como um insumo potencial de uso na agricultura, visto que o teor de água disponível em um dado substrato representa um menor gasto de energia pela planta para aproveitá-la (Vence, 2008).

Pesquisa realizada por Peres et al. (2010) verificou que, na camada de $0-20 \mathrm{~cm}$, houve redução dos valores da umidade volumétrica do solo de 0,103 \% por dia, na condição com palha de cana de açúcar, e de $0,223 \%$ por dia, na condição sem palha, praticamente o dobro daquela verificada na condição de cobertura do solo. Por sua vez, Montenegro et al. (2013), afirmaram que a aplicação de 2 e $4 \mathrm{Mg} \mathrm{ha}^{-1}$ de palha de arroz seca, um substrato com alta relação $\mathrm{C} / \mathrm{N}$, como cobertura morta em um solo arenoso, proporcionou reduções de, respectivamente, $21 \%$ e $51 \%$ no escoamento superficial da água, na menor temperatura e maior umidade do solo. Para Pereira et al. (2015) a utilização de $12 \mathrm{Mg}$ ha-1 ${ }^{-1}$ de cobertura morta de milho proporcionou um armazenamento de $3,62 \mathrm{~mm}$ de água com melhor aproveitamento pela cultura do feijão irrigado e favoreceu a relação soloágua-planta atmosfera por diminuir a temperatura e a 
evaporação da água do solo e aumentar a permanência da umidade no perfil do solo.

A análise de componentes principais $(A C P)$ foi realizada na matriz de dados constituída de 7 variáveis (Figura 4). Quanto ao percentual de variância explicada pelas componentes principais, verifica-se que os dois primeiros componentes principais são responsáveis por $88 \%$ da variabilidade original, sendo que CP1 e CP2 retêm 76,6,2\% e 20,6\%, respectivamente. De acordo com a análise multivariada dos dados obtidos, observou-se variação quanto à eficiência dos tratamentos e foi possível identificar quais variáveis mais se correlacionaram com cada tipo de manejo e os efeitos na alteração nas características do solo.

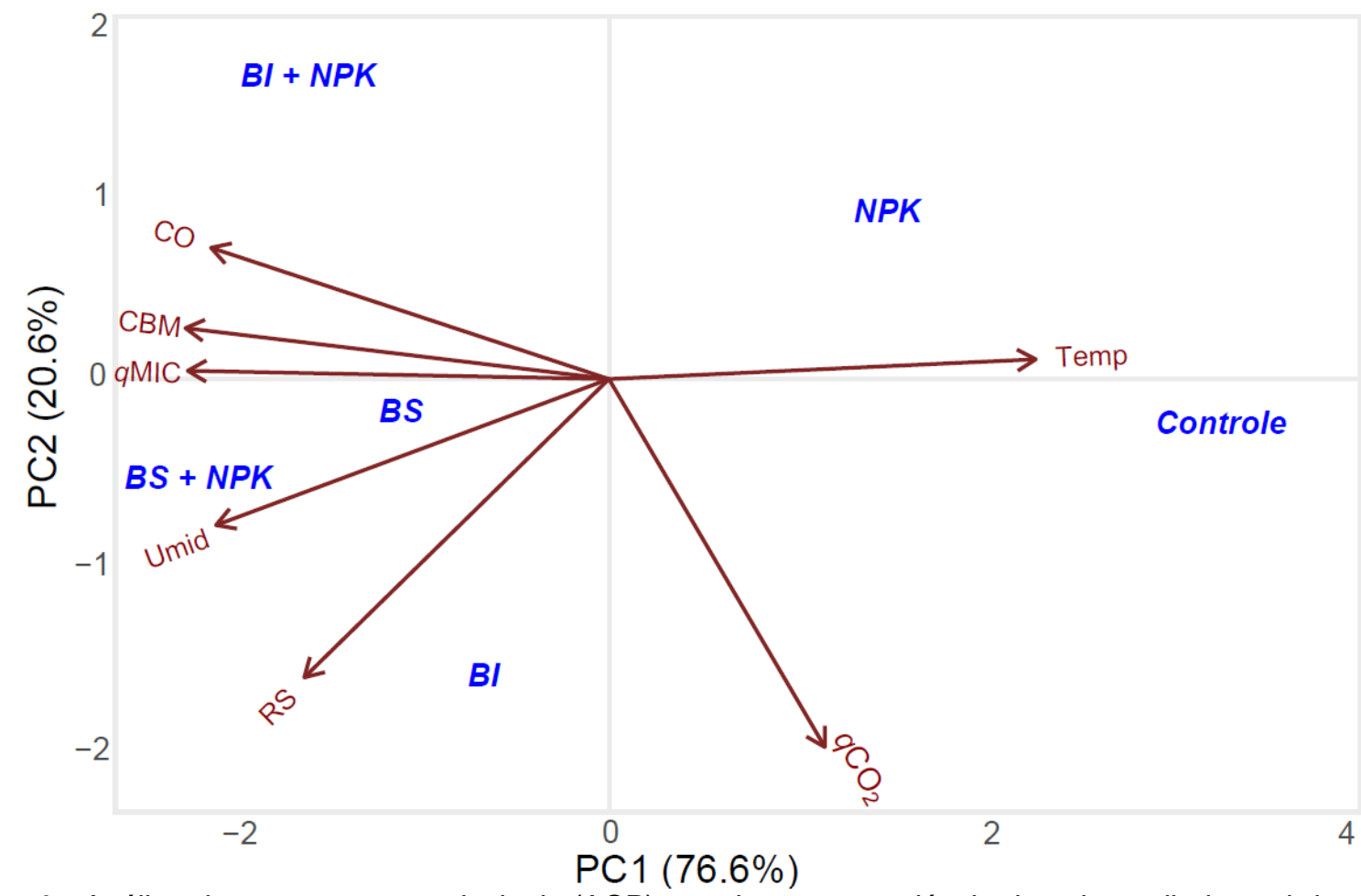

Figura 4 - Análise de componentes principais (ACP) com base nas variáveis do solo avaliadas sob bagana de carnaúba em cobertura ou incorporada e associada ou não a NPK em referência ao solo descoberto e não fertilizado (controle) e uso de adubação mineral com NPK. BI: bagana incorporada; BS: bagana em superfície; todos os tratamentos com bagana receberam $8 \mathrm{t} \mathrm{ha}^{-1}$. Principal component analysis (PCA) based on evaluated soil variables in different treatments in soil cultivated under cover or incorporated carnauba bagasse and associated or not with NPK in reference to uncovered and unfertilized soil (control) and use of fertilization mineral with NPK.

Verificou-se que o aumento da umidade gravimétrica mostrou uma boa correspondência à todas as variáveis estudadas, com exceção do $\mathrm{qCO}_{2}$, enquanto que o aumento da temperatura do solo apresenta efeitos negativos aos atributos microbiológicos do solo, conforme também observado por Rodrigues et al., (2011).

Os tratamentos BS e BS + NPK, estiveram maior relação com o CBM, QMIC, CO e umidade do solo, posicionando-se no quadrante inferior esquerdo. Isto ocorre em função da cera presente nesse resíduo que quando mantido na superfície do solo favorece a reflexão da energia solar evitando a perda de água por evaporação. Além disso, a manutenção de resíduos orgânicos na superfície do solo favorece o aumento do carbono microbiano (Moreira \& Siqueira, 2006). Por sua vez, o tratamento $\mathrm{BI}$ mostrou uma ligeira associação com a respiração e $\mathrm{qCO}_{2}$. Um terceiro grupo foi formado pelos tratamentos com NPK e testemunha, sem cobertura morta, mostraram associação apenas com a temperatura, visto que nesta situação o aumento da temperatura e a diminuição da umidade do solo, levou a uma diminuição nos indicadores microbiológicos, o que pode ser interpretado como resultante da menor taxa de decomposição da matéria orgânica, reduzindo temporariamente a concentração de carbono orgânico e da atividade microbiana.

\section{Conclusão}

O uso da bagana da carnaúba como cobertura morta contribuiu para a manutenção de uma maior umidade e menor temperatura no solo. 
Por meio de análise multivariadas, foi possível verificar que a utilização bagana de carnaúba em superfície isolada ou associada ao NPK mostraram os melhores resultados com relação ao carbono microbiano, quociente microbiano carbono orgânico e umidade do solo, sendo os tratamentos que mais contribuíram para a melhoria da qualidade do solo.

\section{Agradecimentos}

Ao Colégio Técnico de Teresina por ter disponibilizado a área e a logística e aos relatores "ad hoc" do artigo pelas valiosas sugestões e correções.

\section{Referências}

Alvarez V. VH, Novaes RF, Barros NF (1999) Interpretação dos resultados das análises de solos. In Ribeiro AC, Guimarães PTG, Alvarez V. VH (ed) Recomendações para 0 uso de corretivos e fertilizantes em Minas Gerais - 5 $5^{\mathbf{a}}$ Aproximação. Viçosa: Comissão de Fertilidade do Solo do Estado de Minas Gerais (CFSEMG), p. 25-32.

Araújo ASF, Melo WJ (2010) Soil microbial biomass in organic farming system. Ciência Rural, 40(11):24192426.

Araújo ASF, Monteiro RTR (2007) Indicadores biológicos de qualidade do solo. Bioscience Journal, 23(3):66-75.

Bonettl JÁ, Paulino HB, Souza ED, Carneiro MAC, Caetano JO (2018). Soil physical and biological properties in an integrated crop-livestock system in the Brazilian Cerrado. Pesquisa Agropecuária Brasileira, 53(11): 1239-1247.

Brady, NC; Weil RR (2013.) Elementos da natureza e propriedades dos solos. 3.ed. Porto Alegre: Bookman, $704 p$.

Duarte I.B, Gallo AS, Gomes MS, Guimarães, NF, Rocha, DP, Silva, RF (2014) Plantas de cobertura e seus efeitos na biomassa microbiana do solo. Acta Iguazu, 3(2):150-165.

Gasparim E, Ricieri, RP, Silva SL, Dallacort, L, Gnoatto E (2005) Temperatura no perfil do solo utilizando duas densidades de cobertura e solo nu. Acta Scientiarum Agronomy, 27(1): 107-115.

Giacomini SJ, AITA C, Miola ECC, Recous S (2008) Mineralização do carbono da palha de aveia e dejetos de suínos aplicados na superfície ou incorporados ao solo. Revista Brasileira de Ciência Solo, 32(n.spe):2661-2668.

Gomes, JAF, Leite ER, Cavalcante ACR, Cândido MJD, Lempp B, Bomfim MAD, Rogério MCR (2009).
Resíduo agroindustrial da carnaúba como fonte de volumoso para a terminação de ovinos. Pesquisa Agropecuaria.Brasileira, 44(1):58-67.

Gonçalves MPM, Feliciano ALP, Silva AP, Silva LB, Silva KM, Silva J FS (2020) Desenvolvimento em campo de espécies da Caatinga com o uso de resíduo de carnaúba. Brazilian Journal of Development, 6(1):1188-1200.

Gonçalves MPM, Silva MIO, Grugik MA, Feliciano ALP, Silva LB (2019) Substratos alternativos na produção de mudas de Harpalyce brasiliana BENTH. Oecologia Australis, 23(3):464-472.

Islam KR, Weil RR (1998) Microwave irradiation of soil for routine measurement of microbial biomass carbon. Biology and Fertility of Soils, 27(2):408- 416.

Jetter, R, Kunst L (2008) Plant surfasse lipid biosynthetic pathways and their utility for metabolic engineering of waxes and hydrocarbon biofuels. The Plant Journal, 54(4):670-683.

Montenregro AAA, Abrantes JRCB, Lima JLMP, Singh VP, Santos TEM (2013) Impact of mulching on soil and water dynamics under intermittent simulated rainfall. Catena, 109(1):139-149.

Moreira FMS, Siqueira JO (2006) Microbiologia e bioquímica do solo. Lavras: Editora UFLA, 2. Ed., 729 p.

Nogueira DL, Linhares PCF, Silva RIG, Neves AM, Nogueira GSLR, Maracajá, PB, Medeiros AC (2015) Uso exclusivo da palha de carnaúba como condicionador físico do solo no rendimento da cenoura. Informativo técnico do Semiárido, 9(2):64-68.

Oliveira LS, Costa MCG, Sousa HÁ, Blum J, Silva GHA, Abreu MGP, Maia DS (2018) Characterization of Organic Wastes and Effects of Their Application on the Soil. Journal of Agricultural Science, 10(6):291-298.

Perreira FFS, Matsura EE, Mousinho FEP, Bizari DR (2015) Retenção de água em níveis de cobertura morta no feijoeiro irrigado em sistema plantio direto. Irriga, 20(3):557-569.

Peres, JG, Souza CF, Lavorenti NA (2010). Avaliação dos efeitos da cobertura de palha de cana-de-açúcar na umidade e na perda de água do solo. Engenharia Agrícola, 30(5):875-886, 2010.

Queiroga RCF, Nogueira ICC, Bezerra Neto F, Moura ARB, Pedrosa JF (2002) Utilização de diferentes materiais como cobertura morta do solo no cultivo de pimentão. Horticultura Brasileira, 20(3):416- 418. 
R Core Team (2016) Uma Linguagem e Ambiente para Computação Estatística. $\mathrm{R}$ Fundação para Computação Estatística, Viena, Áustria, Disponível em: https://www.-project.org/

Rodrigues HJB, Sá LD, Costa ACL, Silva RB, Moura QL (2011) Variabilidade quantitativa de população microbiana associada às condições microclimáticas observadas em solo de floresta tropical húmida. Revista Brasileira de Meteorologia, 26(4):629-638.

Santoja M, Fernandes C, Gauquelin T, Baldy V. (2015) Climate change effects on litter decomposition: intensive drought leads to a Strong drecrease of litter mixture interactions. Plant Soil, 393(1):69-82.

Silva FL, Campos AO, Santos DA, Oliveira Júnior SD, Padilha CEA, Sousa Junior FC, Macedo GR, Santos ES (2018) Pretreatments of Carnauba (Copernicia prunifera) straw residue for production of cellulolytic enzymes by Trichorderma reesei CCT-2768 by solid state fermentation. Renewable Energy, 116(2):299308.

Sousa RF, Silva RA, Rocha TGF, Santana JAS, Vieira FA (2015) Etnoecologia e etnobotânica da palmeira Carnaúba no Semiárido brasileiro. Cerne, 21(4): $587-$ 594.

Sousa PGR, Sousa JPF, Sousa AM, Costa RNT (2017) Produtividade do mamoeiro cultivado sob aplicação de cinzas vegetais e bagana de carnaúba. Revista Brasileira de Agricultura Irrigada, 11(1):12011212.
Souza AA, Moreira FJC, Araújo BA, Lopes FGN, Silva MES, Carvalho BS (2016) Desenvolvimento inicial de duas variedades de alface em função de dois tipos de substratos e cobertura do solo. Brazilian Journal of Biosystems Engineering, 10(3):316-326.

Souza TEMS, Gonçalves EP, Pereira DS, Santos LM, Machado LS, Souza ER (2018) Redução da erosão em cultivo de sorgo com cobertura morta. Revista Caatinga, 31(3):730-736.

Vieira FF, Dallacor R, Barbieri J D, Dalchiavon FC, Daniel D F (2020) Temperatura e umidade do solo em função do uso de cobertura morta no cultivo de milho. Científica, 48(3):188-199.

Vence LB (2008) Disponibilidad de agua-aire en sustratos para plantas. Ciencia del suelo, 26(2):105114.

Yeomans JC, Bremner JM (1988) A rapid and precise method for routine determination of organic carbon in soil. Communications in soil Science and plant analysis, 19(11):1467-1476.

Ziech ARD, Conceição PC, Luchese AV, Paulus D, Ziech MF (2014) Cultivo de alface em diferentes manejos de cobertura do solo e fontes de adubação. Revista Brasileira de Engenharia Agrícola e Ambiental. 18(9):948-954.

Wang Q, Cao X, Jiang H, Guo Z. (2020) Straw Application and Soil Microbial Biomass Carbon Change: A Meta-Analysis. CLEAN-Soil, Air, Water, v. 49(1):1-7. 